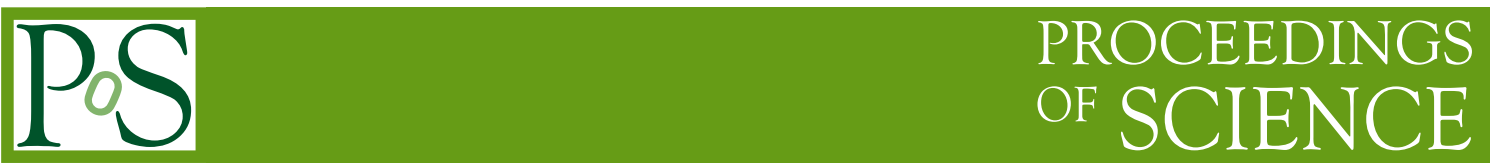

\title{
On the phase diagram of QCD at finite isospin density
}

\section{Philippe de Forcrand}

Institute for Theoretical Physics, ETH Zurich, CH-8093 Zurich, Switzerland

and

CERN, Physics Department, TH Unit, CH-1211 Geneva 23, Switzerland

E-mail: forcrandephys.ethz.ch

\section{Mikhail A. Stephanov}

Physics Department, University of Illinois, 845 W. Taylor St., Chicago, IL 60607-7059, USA

E-mail: misha@uic.edu

\section{Urs Wenger*}

Institute for Theoretical Physics, ETH Zurich, CH-8093 Zurich, Switzerland

E-mail: wenger@phys.ethz.ch

Using a canonical formalism, we determine the equation of state and the phase diagram of eightflavour QCD, as a function of temperature and isospin density. Two mechanisms are at work: Bose condensation of pions at high density, and deconfinement at high temperature. We study their interplay and find that on our small and coarse lattice the first order deconfinement transition appears to end at a critical point at finite density. We investigate the strength of the overlap and of the sign problems and discuss implications for the baryonic density case.

The XXV International Symposium on Lattice Field Theory

July 30 - August 42007

Regensburg, Germany

\footnotetext{
* Speaker.
} 


\section{Introduction}

QCD at finite isospin density is interesting for several reasons. First of all it is a special case of the physically relevant situation where $\mu_{u} \neq \mu_{d} \neq \mu_{s}$. Secondly, it serves as a platform to assess indirectly the limitations of the various numerical approaches to finite baryon density. And thirdly it provides a rich and exciting range of physical phenomena: at low temperature and density there is a pion gas which transforms into a quark gas at high temperature via a deconfinement phase transition, while at low temperature and high density there is Bose condensation of charged pions.

The transition at finite isospin density is, just like in the case of finite baryonic density, due to a large density of a conserved charge, namely the isospin charge $Q$. However, the system does not carry baryon number, but instead the chemical potentials of quarks $u$ and $d$ are set equal in magnitude, but opposite in sign, i.e. $\mu_{u}=-\mu_{d}=\mu_{I} / 2$. Furthermore, taking the $u$ and $d$ quarks masses equal, the system is accessible through lattice simulations because the positivity of the theory is guaranteed by

$$
\tau_{1} \gamma_{5} D \gamma_{5} \tau_{1}=D^{\dagger}
$$

Using this positivity and QCD inequalities, Ref. [1] showed that the symmetry breaking related to the density driven transition must be accompanied by a condensate $\left\langle\bar{\psi} i \gamma_{5} \tau_{1,2} \psi\right\rangle$, i.e. by $\pi^{-} \sim$ $\bar{u} \gamma_{5} d, \pi^{+} \sim \bar{d} \gamma_{5} u$ states. On the lattice, however, this interesting physical system has largely been ignored, with the notable exception of a series of investigations by Kogut and Sinclair [2, 3, 4].

\section{Expectations}

At small isospin densities one can use chiral perturbation theory to describe the physics of the system. The chiral Lagrangian is given by

$$
\mathscr{L}=\frac{1}{4} f_{\pi}^{2} \operatorname{Tr}\left[\mathscr{D}_{\mu} \Sigma \mathscr{D}_{\mu} \Sigma^{\dagger}-2 m_{\pi}^{2} \operatorname{Re} \Sigma\right]
$$

where $\Sigma \in \mathrm{SU}(2)$ is the matrix pion field. The isospin chemical potential $\mu_{I} \tau_{3}$ can be included in $\mathscr{D}_{0}$ at leading order without introducing additional low energy constants and breaks $\mathrm{SU}(2)_{L+R} \rightarrow$ $\mathrm{U}(1)_{L+R}$. The effective potential from the Lagrangian above can be minimised as a function of $\mu_{I}$ using

$$
\bar{\Sigma}=\cos \alpha+i\left(\tau_{1} \cos \phi+\tau_{2} \sin \phi\right) \sin \alpha
$$

where $\phi$ is an irrelevant flavour rotation angle corresponding to the residual $\mathrm{U}(1)_{L+R}$ symmetry and $\alpha$ parametrises the rotation from the standard chiral condensate $\langle\bar{u} u+\bar{d} d\rangle=2\langle\bar{\psi} \psi\rangle_{0}$ into the pion condensate $\left\langle\bar{d} \gamma_{5} u\right\rangle \neq 0$.

From the effective field theory description we can identify two fundamentally different regimes. For $\left|\mu_{I}\right|<m_{\pi}$ no pions can be excited from the vacuum and we have $\bar{\Sigma}=1$, i.e. $\langle\bar{u} u+\bar{d} d\rangle=2\langle\bar{\psi} \psi\rangle_{0}$. This regime corresponds to the standard QCD vacuum at low temperature and zero density.

For $\mu_{I} \geq m_{\pi}, \pi^{+}$particles can be excited from the vacuum and a Bose condensate of $\pi^{+}$forms where $\left\langle\bar{d} \gamma_{5} u\right\rangle \neq 0$. In that regime the remaining $\mathrm{U}(1)_{L+R}$ symmetry is broken spontaneously and hence the transition belongs to the universality class of the $3 d X Y$-model if it is second order.

Finally, for $\mu_{I}>m_{\rho}$ the chiral expansion breaks down and the effective field theory description eq. (2.1) is no longer applicable. Nevertheless, on general ground one can argue [1] that the 

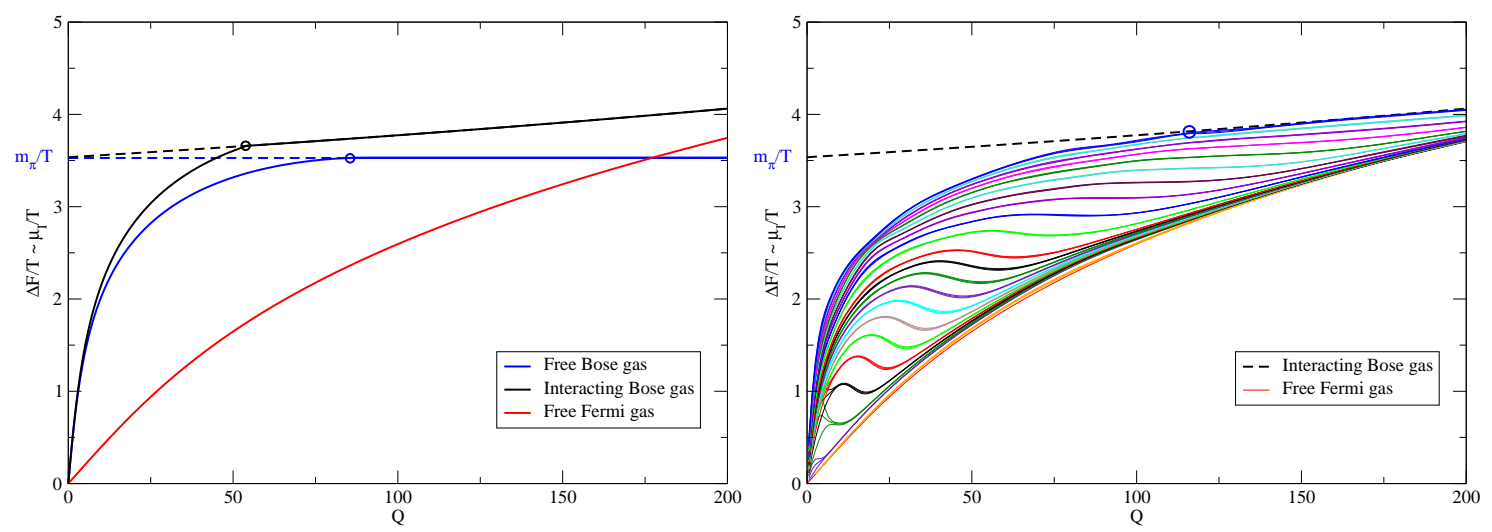

Figure 1: The free energy difference $(F(Q)-F(Q-1)) / T$ versus the number of $u$-quarks $Q$. Left: for a gas of free (blue) and weakly interacting (black) pions and for a gas of free massless fermions (red). Right: simulation results on $8^{3} \times 4$ lattices for successive temperatures, increasing from $\sim \frac{T_{c}}{2}$ to $\sim 1.1 T_{c}$ from top to bottom.

condensate remains $\left\langle\bar{d} \gamma_{5} u\right\rangle \neq 0$ and one might expect a BEC-BCS crossover at large $\mu_{I}$ when the pions "dissociate" (the QCD running coupling becomes weak).

A complete description of the various phases is provided by the equation of state $(\mathrm{EoS})$ of the theory. Here we do not attempt to calculate the full EoS but rather the relation between the isospin density and the chemical potential ${ }^{1}$,

$$
\rho_{I} \equiv \frac{Q}{V}=\rho_{I}\left(\mu_{I}\right)
$$

The canonical free energy $F(Q)=-\ln Z_{C}(Q)$ and its derivative

$$
F(Q)-F(Q-1) \stackrel{V \rightarrow \infty}{\Longrightarrow} \frac{d F}{d \rho_{I}}=\mu_{I}
$$

are numerically well accessible from a canonical formulation of lattice QCD.

The behaviour of the system in the various expected phases can be anticipated using simple semiquantitative considerations. For example, in the confined regime at low temperature and low density, we have a gas of (to a first approximation) free pions. For bosons of mass $m=\hat{m} T$ and a chemical potential $\mu=\hat{\mu} T$, the density reads

$$
\rho_{\text {free }}(\hat{\mu}, \hat{m})=\frac{T^{3}}{2 \pi^{2}} \int_{0}^{+\infty} d \hat{p} \hat{p}^{2}\left(\frac{1}{e^{(\omega-\hat{\mu})}-1}-\frac{1}{e^{(\omega+\hat{\mu})}-1}\right)
$$

where $\omega=\sqrt{\hat{p}^{2}+\hat{m}^{2}}$. The behaviour at zero and low temperature is sketched in Fig. 1 where we plot the free energy difference, eq. (2.4), versus the number of $u$-quarks $Q$. At $\mu_{I}=m_{\pi}$ the system Bose condenses and as a consequence the free energy difference remains constant beyond that point (denoted by the circle in Fig. 1). For an interacting gas of pions the 'EoS' can be described by the effective field theory eq. (2.1) and the density at zero temperature is given by

$$
\rho_{I}=f_{\pi}^{2} \mu_{I}\left(1-\left(\frac{m_{\pi}}{\mu_{I}}\right)^{4}\right)
$$

\footnotetext{
${ }^{1}$ In a slight misuse of notation we will nevertheless refer to eq. (2.3) as EoS in the following.
} 
The interaction leads to an increase of the free energy difference at a given density and temperature as sketched in Fig. 1.

In the high temperature deconfined phase and for quark mass $m_{q} \ll \mu_{I}$ the pressure can be approximated by that of a massless, free Fermi gas, leading to

$$
\frac{\rho_{I}}{T^{3}}=\hat{\mu}_{I}+\frac{1}{\pi^{2}} \hat{\mu}_{I}^{3} .
$$

The corresponding situation is sketched in Fig. 1.

\section{Numerical results}

For computational convenience we consider in our numerical simulations two staggered fermion fields corresponding to $N_{f}=8 \mathrm{QCD}$ in the continuum, i.e. four $u$ - and four $d$-quark species, all degenerate in mass. We keep the lattice volume fixed to $8^{3} \times 4$ as well as the bare quark mass $a m=0.14$ while varying the bare coupling $\beta$ and the isospin chemical potential $a \mu_{I}$. Note that the bare quark mass is tuned in such a way that the deconfinement transition at $T_{c}$ and zero density is first order. Varying $\beta$ changes the lattice spacing $a$ and thereby the temperature $T=\frac{1}{4 a}$ and we cover a range of temperatures between $\frac{1}{2} T_{c} \lesssim T \lesssim T_{c}$. We emphasise that for the range of couplings we consider, the pion mass $a m_{\pi}$, measured at zero temperature, changes very little in units of the lattice spacing, so that the ratio $m_{\pi} / T$ stays almost constant. Note that, contrary to Refs. [2, 3, 4], we do not introduce a $U(1)$-breaking source term in our action. Thus, we can observe the spontaneous symmetry breaking characteristic of Bose condensation without performing any delicate extrapolation. Finally, our results are obtained by combining 68 ensembles at six values of $\mu_{I}$ up to $\mu_{I} / T \lesssim 5$ with Ferrenberg-Swendsen reweighting.

The free energy is obtained from the canonical partition function, $F(Q)=-1 / T \log Z_{C}(Q)$. $Z_{C}(Q)$ is estimated using standard grand canonical Monte Carlo simulations

$$
\frac{Z_{C}(Q)}{Z_{G C}\left(\mu_{I}\right)}=\left\langle\frac{\left|\hat{\operatorname{et}}_{Q}\right|^{2}}{\left|\operatorname{det} D\left(\mu_{I}\right)\right|^{2}}\right\rangle_{Z_{G C}}
$$

where $\operatorname{det}_{Q}$ is obtained by decomposing exactly the part of the measure which depends on $\mu_{I}[5,6]$, i.e., the fermion determinant,

$$
\operatorname{det} D\left(\mu_{I}\right)=\sum_{Q=-3 V}^{+3 V} \operatorname{det} Q \exp \left(Q \mu_{I} / T\right) .
$$

Our results for the free energy difference eq. (2.4) (in units of $T$ ) versus the number of $u$-quarks $Q$ for successive temperatures together with the theoretical expectations are shown in Fig. 1 right. At high temperature (lowest curve) our data is very well described by an ansatz motivated by the free Fermi gas description, eq. (2.7), where the coefficients for the linear and cubic term are multiplied by factors that take into account effects of interactions. The numerical values we obtain are reasonably close to one and consistent with those obtained in [7].

Decreasing the temperature successively, we obtain the second-, third-, ... to-lowest curves. The S-shape of the curves for $T \leq T_{c}$ indicates that $\rho_{I}\left(\mu_{I}\right)$ is multivalued, and is thus characteristic of a first order transition - in the thermodynamic limit the density would jump at the critical isospin 


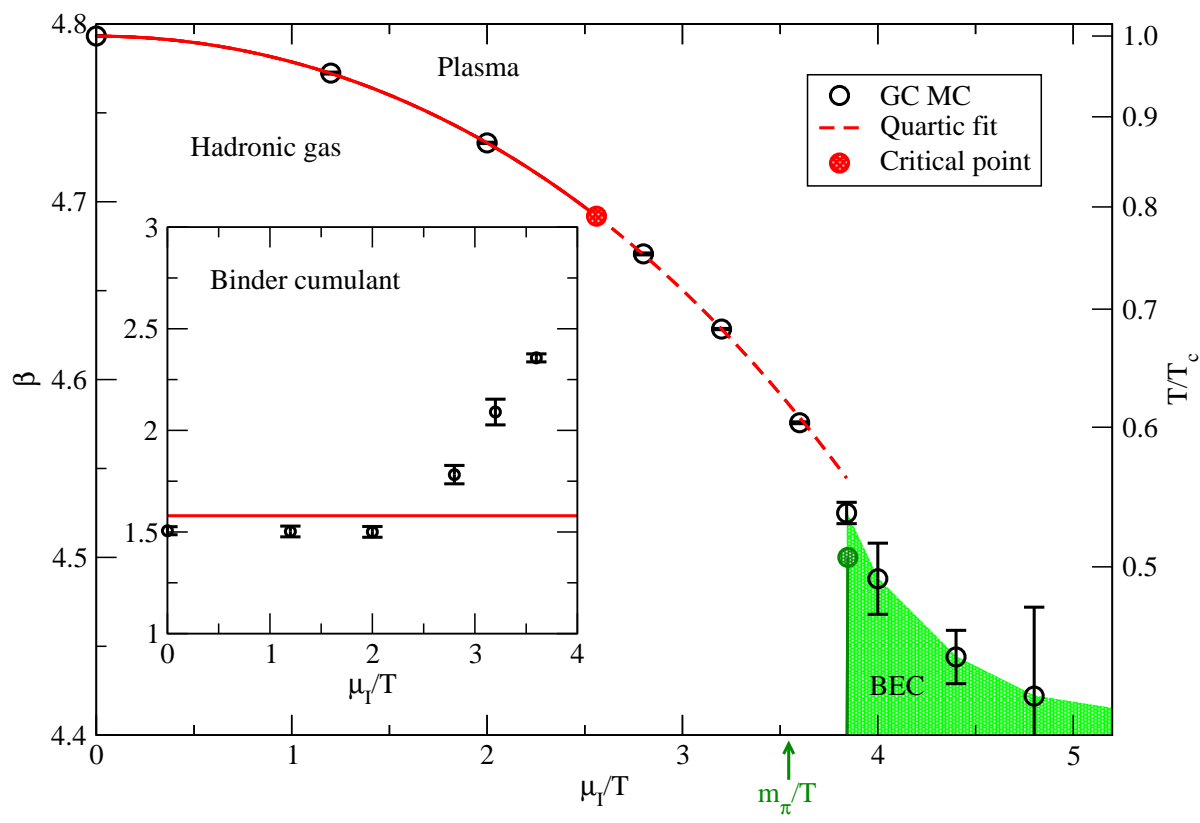

Figure 2: Phase diagram in the $\left(\mu_{I} / T, T / T_{c}\right)$-plane from grand-canonical Monte-Carlo simulations (GCMC) at finite isospin chemical potential. The red circle indicates the critical point as inferred from the change of the behaviour of the Binder cumulants (shown in the inset for the quark condensate) at $\mu_{I} / T \simeq 2.5$. The green circle indicates the transition into the pion condensed phase (BEC) as obtained from the canonical analysis.

chemical potential. Here, the critical $\mu_{I}$ can be determined using the Maxwell construction and it increases as the temperature is decreased. Furthermore, the S-shape becomes smoother and finally disappears at around $T / T_{c} \simeq 0.8$ where the jump in the density vanishes and the first order transition is replaced by a crossover. In order to ascertain the presence of this critical point, we also monitor the distributions of the plaquette, the Polyakov loop, the quark condensate and density by measuring the corresponding Binder cumulants in grand-canonical simulations at fixed $\mu_{I}$. These cumulants remain essentially constant in the region $0 \leq \mu_{I} / T \leq 2$ beyond which they start to grow linearly, cf. inset in Fig. 2. Attributing this behaviour to the critical point of the $3 \mathrm{~d}$ Ising model universality class one can locate the point at $\mu_{I} / T \simeq 2.5$. A finite-size scaling analysis and a continuum extrapolation are of course required to locate this critical point reliably, in particular, to disentangle it from the 2nd order BEC transition. Our findings are similar to those of Ref. [3], which for $N_{f}=3$ also find that the Binder cumulant grows as an isospin chemical potential is on.

Lowering the temperature further, we observe a transition from the pion gas into the Bose condensed phase, marked by a circle in Fig. 1 (right). It turns out that the system can very well be described by the ansatz in eq. (2.6) where the value of $m_{\pi}$ is fixed from our measurement at zero temperature and $f_{\pi}$ is treated as a free parameter.

The full phase diagram in the $\left(\mu_{I} / T, T / T_{c}\right)$-plane is presented in Fig. 2 where the black circles denote our results from the grand-canonical Monte-Carlo simulations (GCMC) and the red circle marks the critical point. For $\mu_{I} \leq m_{\pi}$ the pseudo-critical line between the hadronic gas and the plasma can be well described by a quartic fit (dashed line). The green point marks the entry into 

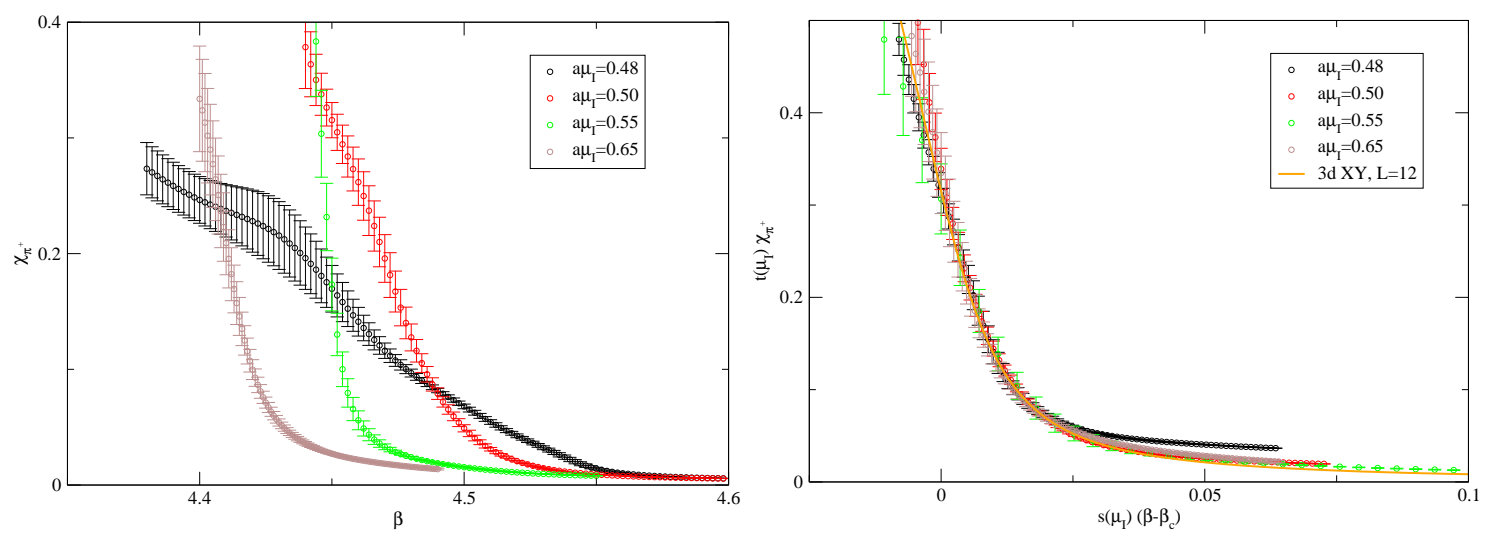

Figure 3: Left: Pion susceptibility $\chi_{\pi^{+}}$for various values of $\mu_{I}$. Right: Data collapse obtained from shifting $\beta$ to $\beta_{c}$ and rescaling both the $x-$ and $y$-axis. Also shown is the universal function from simulations of the $3 d X Y$-model.

the pion condensation phase obtained from Fig. 1 (right). As expected, for $T>0$ this point is shifted towards larger values of $\mu_{I}$ as compared to the zero temperature situation where the transition would occur exactly at $\mu_{I} / T=m_{\pi} / T$ (indicated by the small arrow on the $x$-axis).

The transition from the plasma to the pion condensation phase can be determined by monitoring the pion susceptibility $\chi_{\pi^{+}}=\sum_{x}\left\langle\pi^{+}(0) \pi^{+}(x)\right\rangle$. Since this transition is characterised by the breaking of a $\mathrm{U}(1)$ symmetry, it is expected to belong to the universality class of the $3 d X Y$-model. We can expose the universal behaviour of the transition by comparing the data at different values of $\mu_{I}$ with the universal scaling curve obtained from simulations of the $3 \mathrm{~d} X Y$-model. Fig. 3 shows that the agreement is excellent.

Finally, our calculation also provides a crosscheck of the reweighting method from zero to finite (isospin or baryonic) chemical potential. It turns out that for isospin chemical potential reweighting from $\mu_{I}=0$ is possible, but fails to describe the phase diagram reliably. The overlap of the $\mu_{I}=0$ ensembles with the ones relevant at finite density is not sufficient to determine e.g. the order of the phase transition at finite $\mu_{I}$, and the extrapolation to finite $\mu_{I}$ noticeably underestimates the density of the system. This is understandable from the fact that one tries to extract finite density information from the tail of a density distribution which is generated at $\mu_{I}=0$, i.e. centered at zero.

For an extrapolation to finite baryonic density one has the additional problem that the average sign of the fermionic determinant tends to zero very quickly with $\mu$, as illustrated in Fig. 4 , hence narrowing the range of applicability of the reweighting procedure even further.

\section{Conclusions}

We determined the EoS and the phase diagram of $N_{f}=4+4$ QCD at finite isospin density and finite temperature. The two mechanisms at work are clearly exposed: we observe Bose condensation of pions at high density and deconfinement at high temperature. For our quark mass, the transition from the hadronic gas to the quark-gluon plasma is first order at zero density. As the density is increased, the transition appears to turn into a crossover at $\mu_{I} / T \simeq 2.5$, in qualitative sim- 

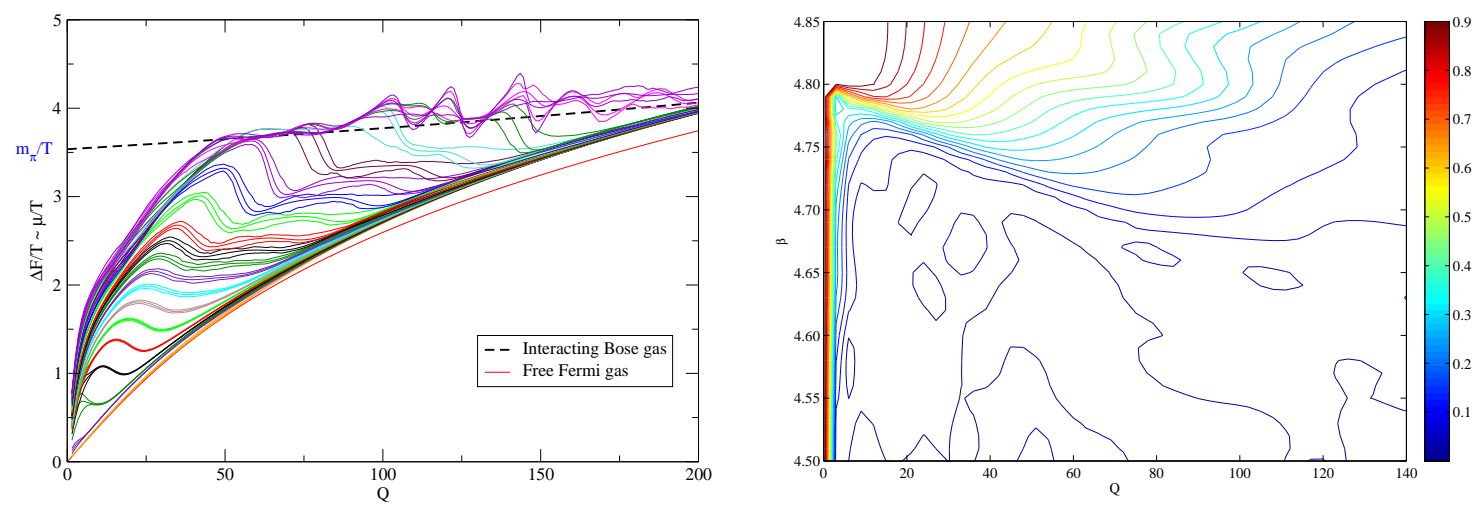

Figure 4: Left: same as Fig. 1 right, but reweighted from zero density simulations only. Right: isocontour lines of the average sign $\left\langle\operatorname{det}_{Q}{ }^{2} /\left|\mathrm{det}_{Q}\right|^{2}\right\rangle$.

ilarity with the results of Ref. [3]. We caution, however, that to confirm these findings a finite-size scaling analysis is still needed.

The transition from the hadronic gas to the BEC phase is determined from a canonical analysis of the free energy density, occurs at low temperature $T / T_{c} \lesssim 0.5$ at $\mu_{I}$ slightly larger than $m_{\pi}$ and is consistent with being second order. The transition from the BEC phase to the plasma phase is also second order and its universal behaviour is confirmed to be that of the $3 d X Y$-model.

Finally, by comparing our (interpolated) results for the $\operatorname{EoS} \rho_{I}\left(\mu_{I}\right)$ with the ones obtained by reweighting from zero to finite $\mu_{I}$, we find that the latter technique systematically underestimates the densities as the chemical potential grows, and that to determine its range of reliability a comparison of different approaches is needed.

\section{Acknowledgements}

We thank Joe Kapusta, Kasper Peeters, Krishna Rajagopal and Dam Son for discussions, the Center for Theoretical Physics, MIT, and the Isaac Newton Institute, Cambridge, for hospitality and the Minnesota Supercomputer Institute for computer resources. M.A.S. is supported, in part, by DOE grant No. DE-FG02-01ER41195.

\section{References}

[1] D. T. Son and M. A. Stephanov, Phys. Rev. Lett. 86 (2001) 592, hep-ph/0005225.

[2] J. B. Kogut and D. K. Sinclair, Phys. Rev. D70 (2004) 094501, hep-lat/0407027.

[3] D. K. Sinclair and J. B. Kogut, PoS LAT2006 (2006) 147, hep-lat/0609041.

[4] D. K. Sinclair and J. B. Kogut, arXiv:0709.2367 [hep-lat].

[5] A. Hasenfratz and D. Toussaint, Nucl. Phys. B371 (1992) 539.

[6] P. de Forcrand and S. Kratochvila, Nucl. Phys. Proc. Suppl. 153 (2006) 62, hep-lat/0602024.

[7] S. Kratochvila and P. de Forcrand, Phys. Rev. D73 (2006) 114512, hep-lat/0602005. 